\title{
Astragaloside IV Inhibits Doxorubicin-Induced Cardiomyocyte Apoptosis Mediated by Mitochondrial Apoptotic Pathway via Activating the PI3K/Akt Pathway
}

\author{
Yuanyuan Jia, Daiying Zuo, Zengqiang Li, Hanmo Liu, Zhengning Dai, Jiayi Cai, \\ Lili Pang, and Yingliang $\mathrm{Wu}^{*}$ \\ Department of Pharmacology, Shenyang Pharmaceutical University; Wenhua Road 103\#, Shenyang 110016, China. \\ Received July 15, 2013; accepted October 2, 2013
}

Doxorubicin (DOX) is a widely used antitumor drug whose application is seriously limited by its cardiotoxicity. Mitochondria-mediated cardiomyocyte apoptosis plays a critical role in DOX-induced cardiotoxicity (DIC). The aim of the present study was to investigate the protective effect of astragaloside IV (3-O-beta-D-xylopyranosyl-6- $O$-beta-D-glucopyranosyl-cycloastragenol, AS-IV), a pure saponin isolated from Astragalus membranaceus, against DOX-induced cardiomyocyte apoptosis in primary cultured neonatal rat cardiomyocytes. Immunocytochemistry and Microculture Tetrazolium (MTT) assays showed that AS-IV significantly reduced DOX-induced cardiomyocyte loss. Additionally, AS-IV markedly ameliorated DOXcaused cardiomyocyte dysfunction via restoring the beating cell ratio and beating rate in cardiomyocytes. Furthermore, AS-IV substantially reduced the mitochondrial reactive oxygen species (ROS) production and lactate dehydrogenase (LDH), creatine kinase-MB isoenzyme (CK-MB) and cytochrome $c$ (CytC) release, and restored the reduced ATP level, succinate dehydrogenase (SDH) and ATP synthase activities induced by DOX, suggesting that AS-IV significantly attenuated DOX-induced mitochondrial damage and dysfunction. It was further observed that DOX-induced cardiomyocyte apoptosis, as qualitatively evaluated by Hoechst 33258 staining and accurately quantified by flow cytometry, was markedly inhibited by AS-IV. Western blot analysis manifested that AS-IV significantly inhibited the activation of mitochondrial apoptotic pathway (MAP) via inducing the phosphorylation of Akt and Bad. Furthermore, phosphatidylinositol 3-kinase (PI3K) inhibitor 2-(4-morpholinyl)-8-phenyl-1(4H)-benzopyran-4-one hydrochloride (LY294002) remarkably inhibited the anti-apoptotic effect of AS-IV. Moreover, AS-IV didn't compromise the antitumor activity of DOX. Taken together, our findings indicate that AS-IV ameliorates DIC, and this beneficial effect appears to be dependent on the activation of the PI3K/Akt pathway. Thus, AS-IV may hold promise as an efficient cardioprotective agent against DIC.

Key words astragaloside IV; doxorubicin; apoptosis; phosphatidylinositol 3-kinase (PI3K); Akt pathway; mitochondrial apoptotic pathway

Doxorubicin (DOX) is a successful antineoplastic agent used to treat a wide range of malignancies. ${ }^{1)}$ Unfortunately, the clinical use of DOX is badly compromised by its cumulative cardiotoxicity leading to irreversible cardiomyopathy and congestive heart failure. ${ }^{2)}$ The precise molecular pathogenesis of DOX-induced cardiotoxicity (DIC) remains highly controversial, although the hypothesis based on oxidative stress has gained the widest favor. ${ }^{3)}$ Furthermore, it should be noted that the treatment of simple antioxidants didn't exhibit much of a prospective protection against DIC. ${ }^{4,5)}$ Hence, several lines of evidence suggested that oxidative stress may be neither the main trigger nor the key executor of DIC, and that increased reactive oxygen species (ROS) formation may be merely a secondary consequence of previous cellular and mitochondrial damage. ${ }^{6,7)}$ At this point, mechanisms other than the traditionally emphasized "oxidative stress hypothesis" might be better bases for seeking and designing approaches against DIC.

Given the limited regenerative capacity of the myocardium, cumulative toxicity may be mainly attributed to the progressive increase of cell loss, and cardiomyocyte apoptosis is widely accepted as a prominent contributor to DIC. ${ }^{8,9)}$ Recently, ever-increasing importance has been attached to the molecular mechanisms of DOX-induced cardiomyocyte apoptosis, and many signal transduction pathways have been

The authors declare no conflict of interest. confirmed involving in this toxicity, including the intrinsic mitochondrial apoptotic pathway (MAP), p53 and mitogenactivated protein kinase (MAPK) pathways. ${ }^{10-12)}$ MAP is considered as one of the overwhelming prominences among these pathways. Mitochondria, the most frequently and progressively damaged sub-cellular organelles of $\mathrm{DOX},{ }^{8)}$ play a key role in DOX-induced cardiomyocyte apoptosis. ${ }^{13)}$ DOX is prone to accumulating in cardiac mitochondria where it enhances ROS production to disrupt mitochondrial bioenergetic and ATP transfer system, resulting in mitochondrial dysfunction, ${ }^{14-16)}$ which is one of the most prominent initiators of MAP. ${ }^{17,18)} \mathrm{Mi}-$ tochondrial dysfunction could activate MAP through inducing Bax translocation from cytosol to mitochondria. Subsequently, the translocation of Bax promotes the release of cytochrome $c$ $(\mathrm{CytC})$ and the consequent formation of apoptosome, resulting in proteolytic cleavage and concomitant activation of caspase-9, which ultimately cleaves and activates caspase-3, leading to apoptotic cell death. Hence, increasing attentions have been paid to the MAP-mediated cardiomyocyte death and the mitochondria-targeted anti-apoptotic mechanisms are increasingly deemed as promising cornerstones for the development of new cytoprotective strategies against DIC.

Astragaloside IV (3-O-beta-D-xylopyranosyl-6- $O$-beta-Dglucopyranosyl-cycloastragenol, AS-IV), isolated from $\mathrm{As}$ tragalus membranaceus, is a bioactive saponin of astragalosides. Accumulating evidence has demonstrated the protective 
effects of AS-IV against cardiac mitochondrial damage. ${ }^{19,20)}$ In addition to these mitochondrial protective effects, AS-IV has also been shown to protect against cardiomyocyte apoptosis. $^{21,22)}$ Taken together, these lines of evidence suggest that AS-IV may possess a mitochondria-targeted anti-apoptotic effect. Li et al. have firstly reported that astragaloside could inhibit DOX-induced cardiomyocyte apoptosis in vivo, ${ }^{23)}$ however, other protective effects of AS-IV, as well as the signaling mechanisms of AS-IV's anti-apoptotic effect against DIC have not been further investigated. Based on these beneficial effects of AS-IV mentioned above, we hypothesized that AS-IV may ameliorate DIC via attenuating the mitochondria damage and dysfunction, as well as inhibiting the cardiomyocyte apoptosis mediated by MAP. To test this hypothesis, primary cultured neonatal rat cardiomyocytes were used to determine the effects of AS-IV on DIC, and the underlying mechanisms of these effects were further investigated.

\section{Experimental}

Reagents and Antibodies The following reagents were used: DOX (Zhejiang Hisun Pharmaceutical Co., Ltd., Zhejiang, China); AS-IV (National Institute for the Control of Pharmaceutical and Biological Products, Beijing, China); MCF-7 human breast cancer cell line (Yanjing Institute of Biotechnology, Shanghai, China); Dulbecco's modified Eagle's medium (DMEM) containing 10\% fetal bovine serum (FBS) (Gibco Invitrogen, Grand Island, NY, U.S.A.); Hoechst 33258, annexin V-fluorescein isothiocyanate (AV-FITC), ethidium bromide (PI) and phosphatidylinositol 3-kinase (PI3K) inhibitor 2-(4-morpholinyl)-8-phenyl-1(4H)-benzopyran-4-one hydrochloride (LY294002) (Beyotime Institute of Biotechnology, Jiangsu, China). The following antibodies were used: anti-Total Akt, anti-phosphorylated-Akt (Ser473) (p-Akt), antiTotal Bad, anti-phosphorylated-Bad (Ser136) (p-Bad), anti- $\alpha$ sarcomeric actin and anti-cardiac Troponin I (cTnI) (Biosynthesis Biotechnology Co., Ltd., Beijing, China); anti-Bcl-2, anti-Bax, anti-CytC, anti-caspase-9, anti-caspase-3 and anti$\beta$-actin (Santa Cruz Biotechnology, Santa Cruz, CA, U.S.A.). The following kits were used: SABC immunocytochemistry kit (Beyotime, Shanghai, China); reactive oxygen species (ROS), lactate dehydrogenase (LDH), creatine kinase-MB isoenzyme (CK-MB), succinate dehydrogenase (SDH), ATP and ATP synthase (Nanjing Jiancheng Bioengineering Institute, Nanjing, China); enhanced chemiluminescence (ECL) detection kit and bicinchoninic acid (BCA) protein assay kit (Beyotime Institute of Biotechnology, Jiangsu, China). The purity of all chemical reagents was at least of the analytical grade.

Cell Culture and Cell Purity Evaluation Primary cultures of cardiomyocytes from neonatal rats and the evaluation of cardiomyocyte purity were performed according to the published methods. ${ }^{24,25)}$

Drug Treatments AS-IV was dissolved in DMEM media as required, and a final concentration of $0.1 \%$ dimethylsulfoxide (DMSO) was proved having no influence on normally cultured cardiomyocytes (data were not provided). In no cases were the final concentrations of DMSO added to cells more than $0.1 \%$ throughout the entire study. Normal culture (DMEM with $0.1 \% \mathrm{DMSO}, 37^{\circ} \mathrm{C}$ ) was served in the Control group. Cells in the AS-IV groups were only treated with different concentrations of AS-IV $(3.75,7.5,15,30,60 \mu \mathrm{g} / \mathrm{mL})$ for $24 \mathrm{~h}$. Cells in the DOX group were only treated with DOX
$(1 \mu \mathrm{M})$ for $24 \mathrm{~h}^{26)}$ In the rest groups, cells were all treated with DOX $(1 \mu \mathrm{M})$ for $24 \mathrm{~h}$. Prior to DOX treatments, cells were incubated with various concentrations of AS-IV (3.75, 7.5, $15,30,60 \mu \mathrm{g} / \mathrm{mL}$ ) for $1 \mathrm{~h}$ in the DOX+AS-IV groups. In the DOX+AS-IV+LY294002 group, cells were treated with both LY294002 $(10 \mu \mathrm{M})$ and AS-IV $(30 \mu \mathrm{g} / \mathrm{mL}) 1 \mathrm{~h}$ before DOX treatment. Cells in the DOX+LY294002 group were treated with LY294002 $(10 \mu \mathrm{M}) 1 \mathrm{~h}$ before DOX treatment.

Determination of Cell Survival The cell survival was qualitatively evaluated by immunocytochemistry and accurately quantified via Microculture Tetrazolium (MTT) assay. The immunocytochemistry was performed using a SABC immunocytochemistry kit. Anti-cTnI was used as the primary antibody and horseradish peroxidase-conjugated immunoglobulin $\mathrm{G}(\mathrm{IgG})$ was used as the secondary antibody. The MTT assay was performed according to a published method. ${ }^{27)}$ Each experiment was performed at least in triplicate.

Determination of Cell Function The cell function was evaluated by determining the beating cell ratio and beating rate in cardiomyocytes using an inverted phase-contrast microscope in a chamber controlled at $37^{\circ} \mathrm{C}$. Twenty-four hours after treatments, the beating cell number in 100 randomly selected cardiomyocytes was counted in each well, and the ratio of beating cell number to total cell number (100) was deemed as the beating cell ratio. The beating cell ratios of six wells were summarized in each group. The beating rates (beating number per minute, bpm) of three randomly selected beating cardiomyocytes were counted in each well and the beating rates of six wells were summarized in each group. Each experiment was performed at least three times by using separately prepared cell cultures.

Determination of Mitochondrial Damage and Dysfunction in Cardiomyocytes The probe 6-carboxy-2',7'-dichlorodihydrofluorescein diacetate (6-carboxy- $\mathrm{H}_{2}$ DCFDA) was used to measure mitochondrial ROS. It is a non-fluorescent and cell permeable analog that is oxidized to highly fluorescent carboxy-dichlorofluorescein (carboxy-DCF) as measured at Ex $488 \mathrm{~nm} / \mathrm{Em} 520 \mathrm{~nm}$ with fluorescence microscopy and expressed in arbitrary units (a.u.). Cells were incubated with carboxy- $\mathrm{H}_{2} \mathrm{DCFDA}(1 \mu \mathrm{M})$ at $37^{\circ} \mathrm{C}$ for $15 \mathrm{~min}$, and washed with D-Hanks buffer before mitochondria extraction. The fluorescence intensity was quantified using MetaMorph software. Each experiment was performed at least in triplicate.

The mitochondrial damage was evaluated via measuring the activities of $\mathrm{LDH}$ and CK-MB in mitochondrial supernatant according to the kits' instructions. The mitochondrial dysfunction was determined by examining the SDH and ATP synthase activities as well as the ATP level in mitochondrial homogenate according to the kits' instructions. Each experiment was performed at least in triplicate.

Hoechst 33258 Staining Hoechst 33258 staining was used to identify the morphological features of apoptosis according to a published method. ${ }^{27)}$ Each experiment was performed at least in triplicate.

Flow Cytometry Flow cytometry was used to accurately determine the cell apoptosis rate. Apoptotic cells were differentiated from viable or necrotic ones by combined application of AV-FITC and PI. The samples were washed twice and adjusted to a concentration of $1 \times 10^{6}$ cells $/ \mathrm{mL}$ with cold $\mathrm{D}$ Hanks buffer. Following that, AV-FITC $(10 \mu \mathrm{L})$ and PI $(10 \mu \mathrm{L})$ were added into $100 \mu \mathrm{L}$ of cell suspension, which was incubat- 
ed for $15 \mathrm{~min}$ at room temperature in the dark. Finally, $400 \mu \mathrm{L}$ of binding buffer was added to each sample without washing and analyzed using flow cytometry (FCM). Each experiment was performed at least in triplicate.

Western Blot Analysis The expression levels of proteins associated with the MAP and PI3K/Akt pathway were detected by Western blot analysis according to a published method. ${ }^{26)}$ Briefly, total protein extracts of each group cells were resolved by $12 \%$ sodium dodecyl sulfate-polyacrylamide gel electrophoresis (SDS-PAGE) and transferred to polyvinylidene difluoride (PVDF) membranes. After blocking, the PVDF membranes were washed 3 times for $15 \mathrm{~min}$ with TBST at room temperature and incubated with the anti-Total Akt, anti-p-Akt (Ser473), anti-Total Bad, anti-p-Bad (Ser136), antiBcl-2, anti-Bax, anti-CytC, anti-caspase-9, anti-caspase-3 and anti- $\beta$-actin antibodies overnight at $4^{\circ} \mathrm{C}$. Following extensive washing, membranes were incubated with peroxidase-linked secondary antibodies for $2 \mathrm{~h}$. After washing three times for 15 min with TBST at room temperature once more, the immunoreactivity was visualized by ECL, and membranes were exposed to Kodak films. western blot results were quantified with Wright Cell Imaging Facility (WCIF) Image J version 1.37c. Each experiment was performed at least in triplicate.

Interference of AS-IV on the Antitumor Activity of DOX MCF-7 human breast cancer cell line was used to check the interference of AS-IV on the antitumor activity of DOX. The cancer cells were seeded into 96-well plates at a starting density of $2.5 \times 10^{4}$ cells/well and cultured overnight in a DMEM medium containing $10 \%$ fetal calf serum at $37^{\circ} \mathrm{C}$ humidified with $5 \% \mathrm{CO}_{2}$. Normal culture (DMEM with $0.1 \%$ DMSO, $37^{\circ} \mathrm{C}$ ) was served in the Control group. Cells in the DOX group were treated with DOX $(10 \mu \mathrm{M})$ for $24 \mathrm{~h}$. Cells in the DOX+AS-IV groups were treated with DOX $(10 \mu \mathrm{M})$ and AS-IV $(15,30 \mu \mathrm{g} / \mathrm{mL})$ for $24 \mathrm{~h}$. Twenty-four hours later, MTT assay was used to assess the viability of cancer cells. Each experiment was performed at least in triplicate.

Statistical Analysis All experiments were run in at least 6 parallels and repeated 3 times. All statistical analyses were performed using SPSS 13.0 software (SPSS Inc., Chicago, IL, U.S.A.). Data obtained from three independent experiments were expressed as mean \pm S.D. Multiple comparisons among different treatment groups were evaluated by one-way ANOVA followed by Tukey test as post hoc analysis. Differences between groups were considered significant at $p<0.05$.

\section{Results}

Cardiomyocyte Purity Evaluation In this study, the probable purity range of primary cultured cardiomyocytes was $95.2 \pm 3.4$ to $96.1 \pm 3.8 \%$, as provided in supplementary Table 1 .

AS-IV Attenuated DOX-induced Cytotoxicity in Cardiomyocytes Firstly, the cell survival was identified by immunocytochemistry via detecting the cTnI expression in cardiomyocytes (Fig. 1). The positive cTnI expression cells, which were stained brown in cytoplasm, were deemed as viable cells. The results, as shown in Fig. 1A, indicated that most of the cardiomyocytes in the Control group were viable. DOX evidently reduced the number of viable cells compared with the Control group (Fig. 1B). Cells pretreated with AS-IV (15, $30 \mu \mathrm{g} / \mathrm{mL}$ ) showed obvious increases in the number of viable cells compared with the DOX group (Figs. 1C, D).

Subsequently, the cell viability was assessed by MTT assay to accurately quantify the cell survival. As shown in Fig. $2 \mathrm{~A}$, there were no significant differences in the cell viability between the Control and AS-IV groups. As shown in Fig. 2B, cells treated with DOX $(1 \mu \mathrm{M})$ for 24 to $48 \mathrm{~h}$ showed significant decreases in the cell viability in a time-dependent manner,
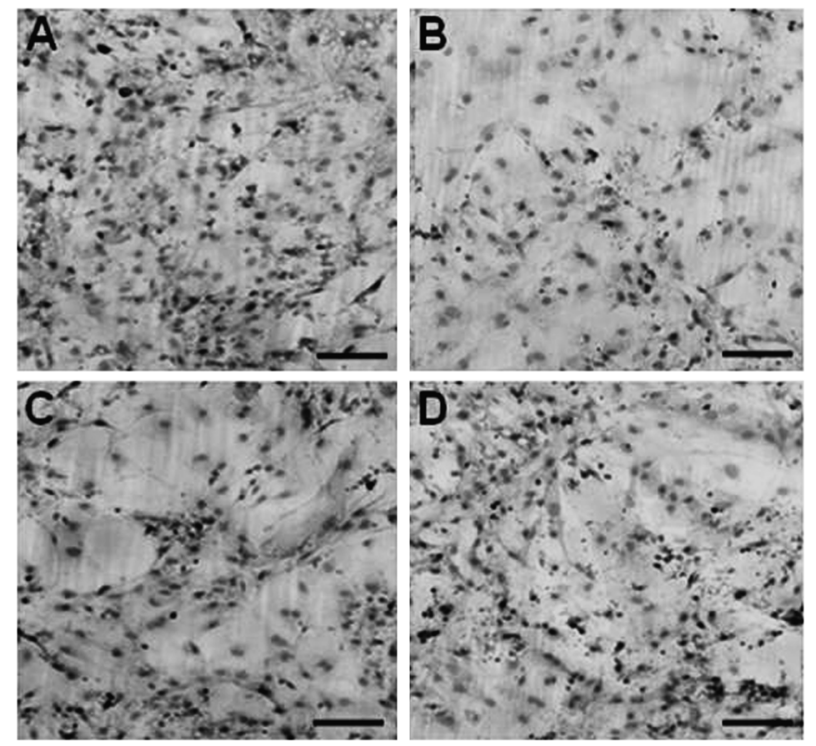

Fig. 1. Effect of AS-IV on the cTnI Expression in DOX-Treated Cardiomyocytes (Immunocytochemistry)

(A) Control group. (B) DOX $(1 \mu \mathrm{M})$ group. (C) DOX $(1 \mu \mathrm{M})+\mathrm{AS}-\mathrm{IV}(15 \mu \mathrm{g} / \mathrm{mL})$ group. (D) DOX $(1 \mu \mathrm{m})+$ AS-IV $(30 \mu \mathrm{g} / \mathrm{mL})$ group. Scale bar, $200 \mu \mathrm{m}$. All experiments were repeated at least three times.
A

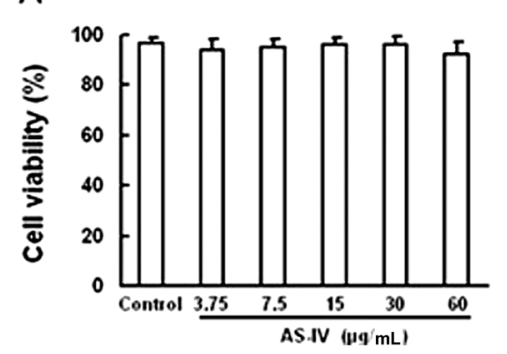

B

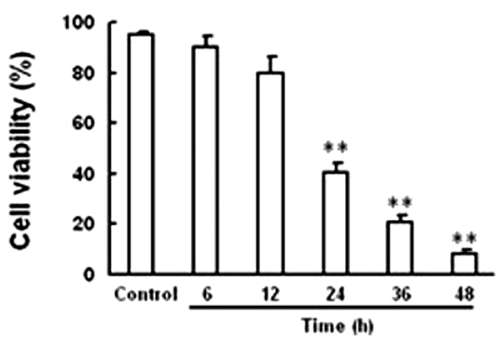

C

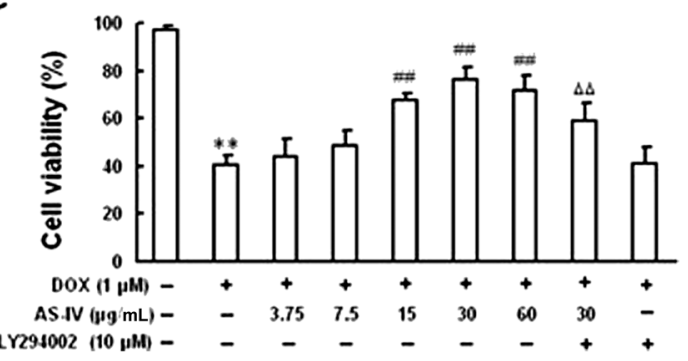

Fig. 2. Effect of AS-IV on DOX-Induced Cardiomyocyte Cytotoxicity (MTT Assay)

(A) Effect of AS-IV treatment on the cell viability in normally cultured cardiomyocytes. Cardiomyocytes were treated with different concentrations of AS-IV (3.75, 7.5, $15,30,60 \mu \mathrm{g} / \mathrm{mL}$ ) for $24 \mathrm{~h}$. (B) Time-dependent toxic effect of DOX on cardiomyocytes viability. Cardiomyocytes were treated with DOX (1 $\mu \mathrm{M})$ for 6 to $48 \mathrm{~h}$. (C) Effects of AS-IV and co-administration of AS-IV with LY294002 on DOX-induced cardiomyocytes loss. The concentrations of AS-IV were from 3.75 to $60 \mu \mathrm{g} / \mathrm{mL}$. The concentration of LY294002 was $10 \mu \mathrm{M}$. Data are presented as mean \pm S.D. ${ }^{* *} p<0.01 v s$. Control group; ${ }^{\# \#} p<0.01 v s$. DOX $(1 \mu \mathrm{M})$ group; ${ }^{\Delta} p<0.01 v s$. DOX $(1 \mu \mathrm{M})+\mathrm{AS}-\mathrm{IV}(30 \mu \mathrm{g} / \mathrm{mL})$ group. All experiments were repeated at least three times. 
as compared with the Control group $(p<0.01)$. As illustrated in Fig. 2C, the cell viability in the Control group was 97.1土 $2.0 \%$. DOX $(1 \mu \mathrm{M})$ treatment for $24 \mathrm{~h}$ induced a significant decrease in the cell viability compared with the Control group $(40.4 \pm 4.1 \%, p<0.01)$. In contrast, AS-IV $(15,30 \mu \mathrm{g} / \mathrm{mL})$ pretreatments significantly prevented cardiomyocytes from DOX-induced damage in a dose-dependent manner, as compared with the DOX group $(67.5 \pm 3.2,76.3 \pm 5.1 \%$, respectively, $p<0.01$ ). However, LY294002 treatment (PI3K inhibitor, $10 \mu \mathrm{M})$ markedly reduced the cell viability restored by AS-IV, as compared with the DOX+AS-IV $(30 \mu \mathrm{g} / \mathrm{mL})$ group $(41.3 \pm$ $6.6 \%, p<0.01)$. There were no significant differences in the cell viability between the DOX and DOX+LY294002 groups.

The cell function was determined by measuring the beating cell ratio and beating rate in cardiomyocytes. The results, as shown in supplementary Table 2, indicated that the beating cell ratio and beating rate were both at their normal levels in the cardiomyocytes of the Control group (96 $2 \%$ and $67.8 \pm$ $6.2 \mathrm{bpm}$, respectively). DOX reduced the beating cell ratio and beating rate in cardiomyocytes to $66 \pm 9 \%$ and $37.8 \pm 5.5 \mathrm{bpm}$, respectively, as compared with the Control group $(p<0.01)$. In contrast, AS-IV $(15,30 \mu \mathrm{g} / \mathrm{mL})$ pretreatments induced significant increases in the beating cell ratio and beating rate in DOX-treated cardiomyocytes in a dose-dependent manner, as compared with the DOX group $(p<0.05$ and $p<0.01$, respectively). The beating cell ratios and beating rates in the cardiomyocytes of DOX+AS-IV $(15 \mu \mathrm{g} / \mathrm{mL})$ and DOX+AS-IV $(30 \mu \mathrm{g} / \mathrm{mL})$ groups were $79 \pm 11 \%$ and $50.1 \pm 4.8 \mathrm{bpm}$, and $88 \pm$ $9 \%$ and $62.5 \pm 5.4 \mathrm{bpm}$, respectively.
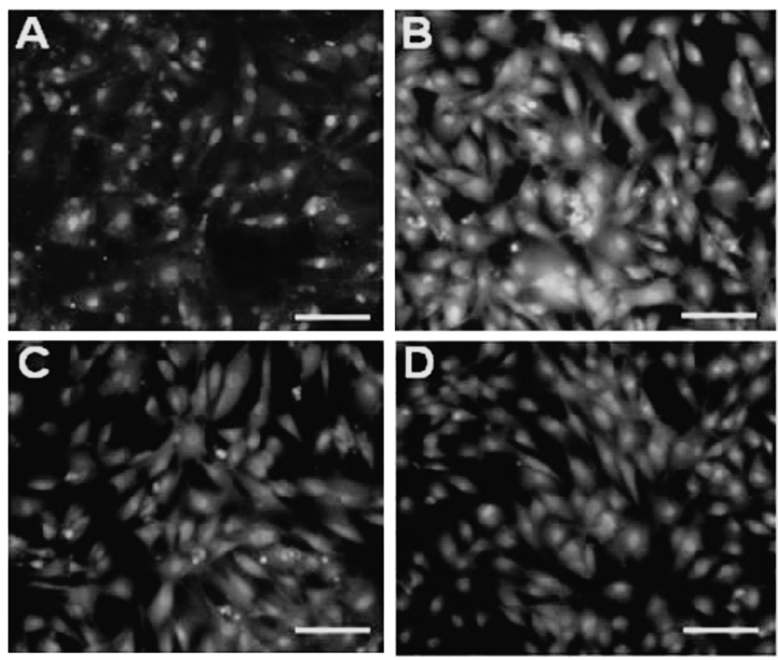

E

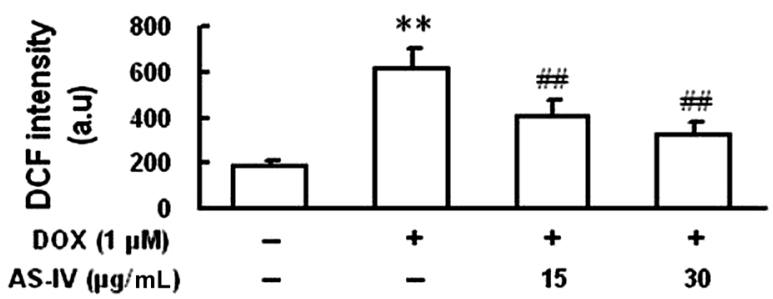

Fig. 3. Effect of AS-IV on DOX-Induced ROS Production (6-(Carboxy$\mathrm{H}_{2}$ DCFDA Fluorescent Probe Assay)

(A) Control group. (B) DOX $(1 \mu \mathrm{M})$ group. (C) DOX $(1 \mu \mathrm{M})+\mathrm{AS}-\mathrm{IV}(15 \mu \mathrm{g} / \mathrm{mL})$ group. (D) DOX $(1 \mu \mathrm{m})+$ AS-IV $(30 \mu \mathrm{g} / \mathrm{mL})$ group. Scale bar, $50 \mu \mathrm{m}$. (E) AS-IV reduced the increase in carboxy-DCF fluorescence intensity induced by DOX. Data are presented as mean \pm S.D. $* * p<0.01 v s$. Control group; ${ }^{\#} p<0.01 v s$. DOX $(1 \mu \mathrm{M})$ group. All experiments were repeated at least three times.
AS-IV Attenuated DOX-Induced Mitochondrial Damage and Dysfunction The probe 6-carboxy- $\mathrm{H}_{2}$ DCFDA was used to determine the mitochondrial ROS level. As shown in Fig. 3A, low fluorescence intensity was seen in the cells of the Control group. DOX obviously enhanced the fluorescence intensity compared with the Control group (Fig. 3B). However, $\operatorname{AS}-\mathrm{IV}(15,30 \mu \mathrm{g} / \mathrm{mL})$ pretreatments evidently attenuated the fluorescence intensity enhanced by DOX (Figs. 3C,D). The ROS levels were summarized by quantifying the carboxy-DCF levels to make a direct comparison (Fig. 3E). As shown in Fig. 3E, the carboxy-DCF intensity was $188 \pm 25$ (a.u.) in the Control group. DOX substantially increased the carboxy-DCF intensity to $613 \pm 88$ (a.u.), as compared with the Control group $(p<0.01)$. In contrast, AS-IV $(15,30 \mu \mathrm{g} / \mathrm{mL})$ pretreatments significantly reduced DOX-increased carboxy-DCF intensity in a dose-dependent manner, as compared with the DOX group $(p<0.01)$. The carboxy-DCF intensities in the DOX+AS-IV $(15 \mu \mathrm{g} / \mathrm{mL})$ and DOX + AS-IV $(30 \mu \mathrm{g} / \mathrm{mL})$ groups were $402 \pm 74$ and $320 \pm 61$ (a.u.), respectively.

The mitochondrial damage and dysfunction were evaluated via determining their associated marker levels/activities using biochemical kits. As shown in supplementary Table 3, DOX significantly increased the LDH and CK-MB activities in mitochondrial supernatant, and markedly reduced the SDH and ATP synthase activities and ATP level in mitochondrial homogenate, as compared with the Control group $(p<0.01)$. In contrast, AS-IV $(15,30 \mu \mathrm{g} / \mathrm{mL})$ pretreatments significantly reduced the $\mathrm{LDH}$ and $\mathrm{CK}-\mathrm{MB}$ activities, and preserved the SDH and ATP synthase activities and ATP level in a dose-dependent manner, as compared with the DOX group $(p<0.01)$. Furthermore, $\operatorname{AS}-\mathrm{IV}(15,30 \mu \mathrm{g} / \mathrm{mL})$ treatments alone markedly elevated the ATP synthase activity and ATP level in a dose-dependent manner, as compared with the Control group $(p<0.05$ or $p<0.01)$.

AS-IV Attenuated DOX-Induced Cardiomyocyte Apoptosis The morphological changes of apoptotic cells were indentified by Hoechst 33258 staining, as illustrated in Fig. 4. The cells in the Control group exhibited intact cell nuclei (Fig. 4A). While DOX-treated cardiomyocytes featured typical characteristics of apoptosis, including the shrinkage and fragmentation of nuclei and even the appearance of a few apoptotic bodies (Fig. 4B). In contrast, AS-IV pretreatments evidently attenuated the apoptotic changes induced by DOX (Figs. 4C,D). However, LY294002 treatment (10 $\mu \mathrm{M})$ obviously reversed AS-IV-alleviated apoptotic changes (Fig. 4E).

Cardiomyocytes were stained with PI and AV-FITC to accurately quantify the apoptosis rate via flow cytometry (Fig. 5). The cardiomyocyte apoptosis rates were provided in Fig. 5F. The cardiomyocyte apoptosis rate in the Control group was $1.7 \pm 0.3 \%$ (Fig. 5A). DOX induced significant apoptotic damage in cardiomyocytes compared with the Control group (43.3 $\pm 6.2 \%, p<0.01$, Fig. 5B). While AS-IV $(15,30 \mu \mathrm{g} / \mathrm{mL})$ pretreatments significantly reduced DOX-increased apoptosis rate in a dose-dependent manner, as compared with the DOX group $(26.8 \pm 6.5,18.7 \pm 3.7 \%$, respectively, $p<0.01$, Figs. 5C,D). However, LY294002 treatment significantly increased AS-IV-reduced apoptosis rate to $39.6 \pm 5.8 \%$, as compared with the DOX+AS-IV $(30 \mu \mathrm{g} / \mathrm{mL})$ group $(p<0.01$, Fig. $5 \mathrm{E})$.

AS-IV Activated the PI3K/Akt Pathway to Inhibit the Cardiomyocyte Apoptosis Mediated by MAP The expression levels of proteins associated with the MAP were detected 

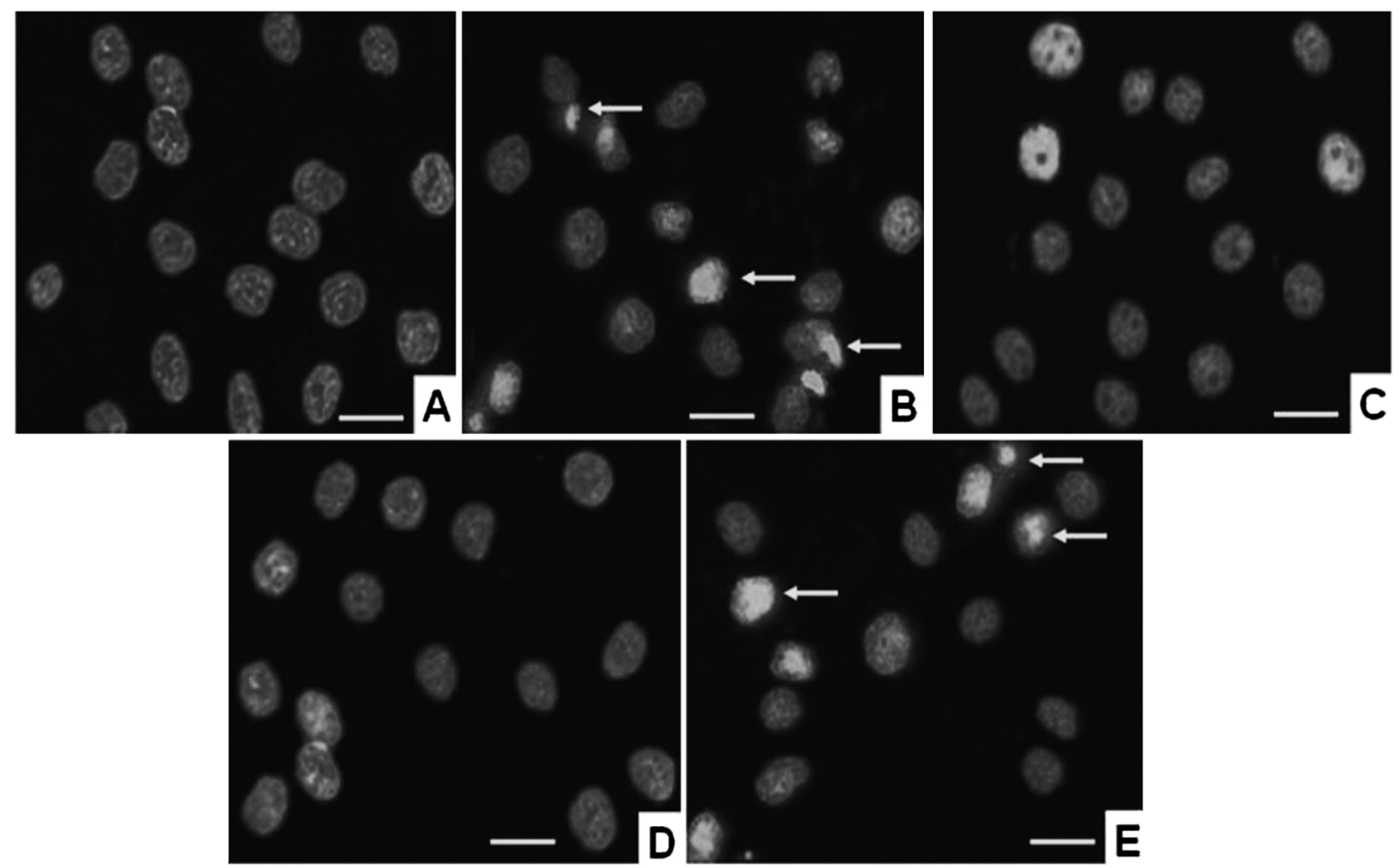

Fig. 4. Effect of AS-IV on DOX-Induced Cardiomyocyte Apoptosis (Hoechst 33258 Staining)

(A) Control group. (B) DOX $(1 \mu \mathrm{M})$ group. (C) DOX $(1 \mu \mathrm{M})+\mathrm{AS}-\mathrm{IV}(15 \mu \mathrm{g} / \mathrm{mL})$ group. (D) DOX $(1 \mu \mathrm{m})+\mathrm{AS}-\mathrm{IV}(30 \mu \mathrm{g} / \mathrm{mL})$ group. (E) DOX $(1 \mu \mathrm{m})+\mathrm{AS}-\mathrm{IV}(30 \mu \mathrm{g} /$ $\mathrm{mL})+\mathrm{LY} 294002(10 \mu \mathrm{M})$ group. Scale bar, $5 \mu \mathrm{m}$. All experiments were repeated at least three times.
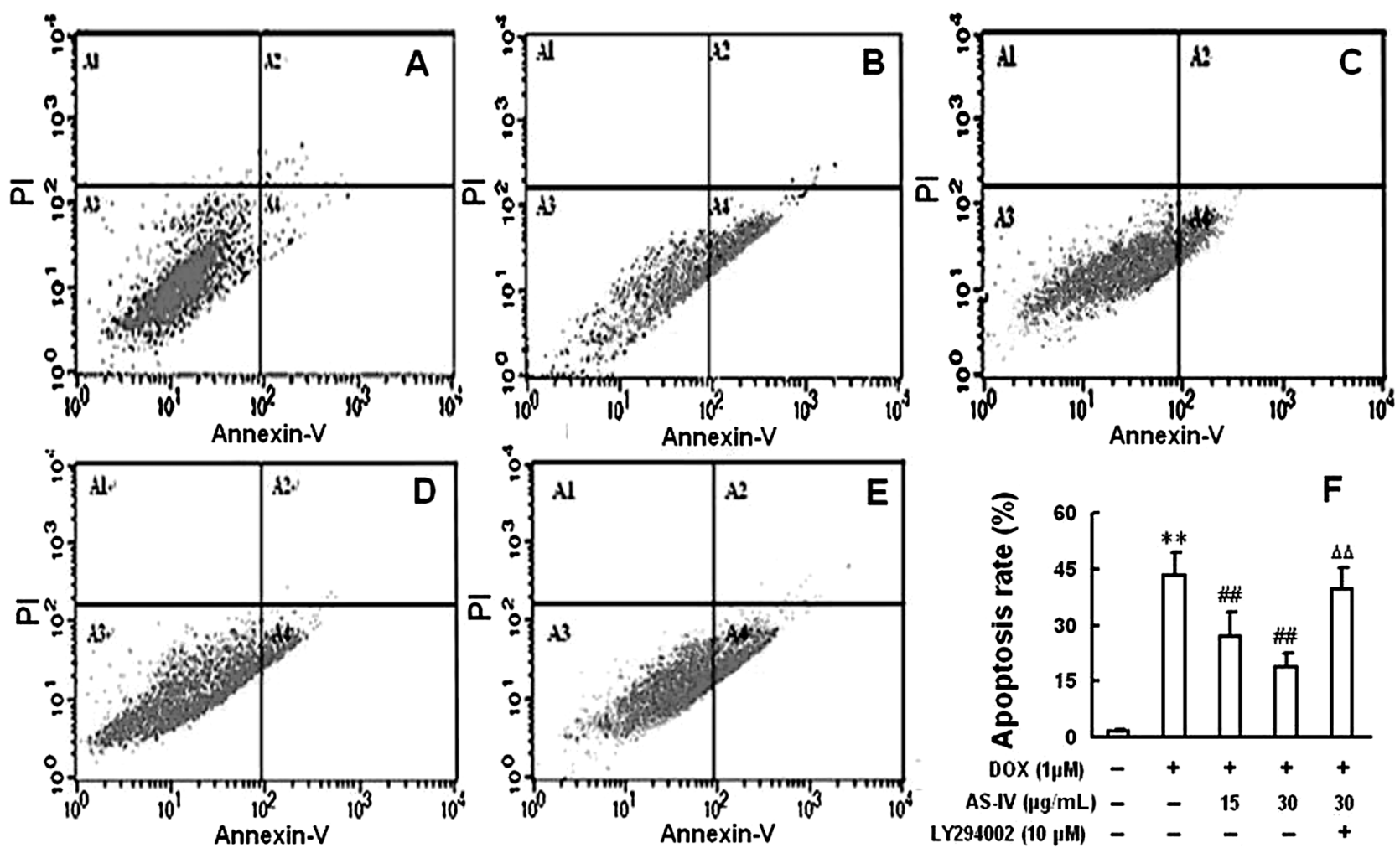

Fig. 5. Effect of AS-IV on DOX-Induced Cardiomyocyte Apoptosis (Flow Cytometry Assay)

(A) Control group. (B) DOX $(1 \mu \mathrm{M})$ group. (C) DOX $(1 \mu \mathrm{M})+$ AS-IV $(15 \mu \mathrm{g} / \mathrm{mL})$ group. (D) DOX $(1 \mu \mathrm{M})+\mathrm{AS}-\mathrm{IV}(30 \mu \mathrm{g} / \mathrm{mL})$ group. (E) DOX $(1 \mu \mathrm{M})+\mathrm{AS}-\mathrm{IV}$ $(30 \mu \mathrm{g} / \mathrm{mL})+\mathrm{LY} 294002(10 \mu \mathrm{M})$ group. (F) AS-IV reduced the increase in cardiomyocyte apoptosis rate induced by DOX. Data are presented as mean \pm S.D. $* * p<0.01 v s$. Control group; ${ }^{\#} p<0.01$ vs. DOX $(1 \mu \mathrm{m})$ group; ${ }^{\Delta \Delta} p<0.01 v$ s. DOX $(1 \mu \mathrm{M})+\mathrm{AS}-\mathrm{IV}(30 \mu \mathrm{g} / \mathrm{mL})$ group. All experiments were repeated at least three times.

by Western blot analysis (Fig. 6). As illustrated in Fig. 6A, DOX significantly reduced the expression of $\mathrm{Bcl}-2$ protein, whereas markedly enhanced the expression of Bax protein and promoted the release of $\mathrm{CytC}$, as compared with the Control group $(p<0.01)$. In addition, DOX significantly enhanced the expressions of caspase- 9 and caspase- 3 proteins via inducing the proteolysis of pro-caspase- 9 and pro-caspase- 3 , respectively, as compared with the Control group $(p<0.01$, Fig. 6B). In contrast, AS-IV significantly enhanced the expression of Bcl-2 protein, whereas markedly reduced the expression of Bax 
A

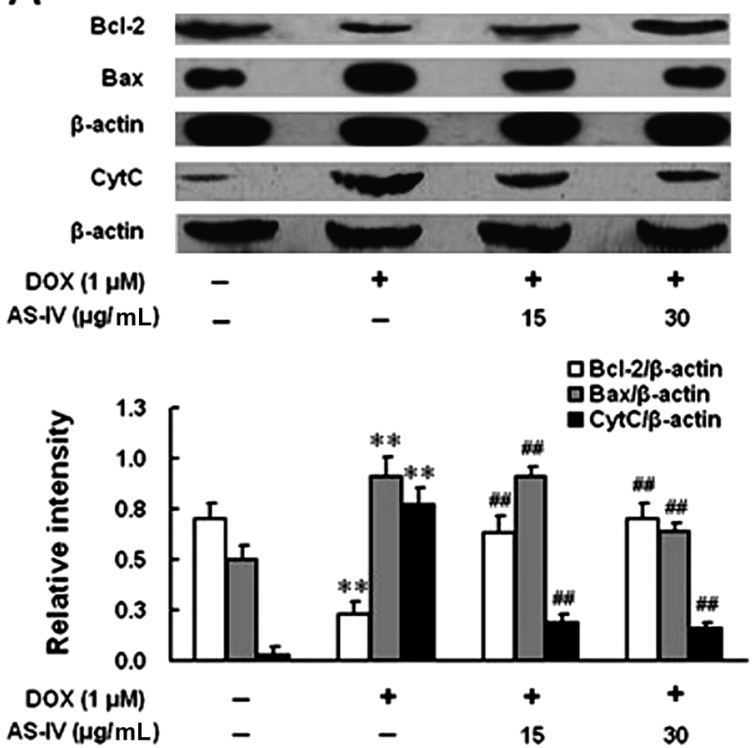

B

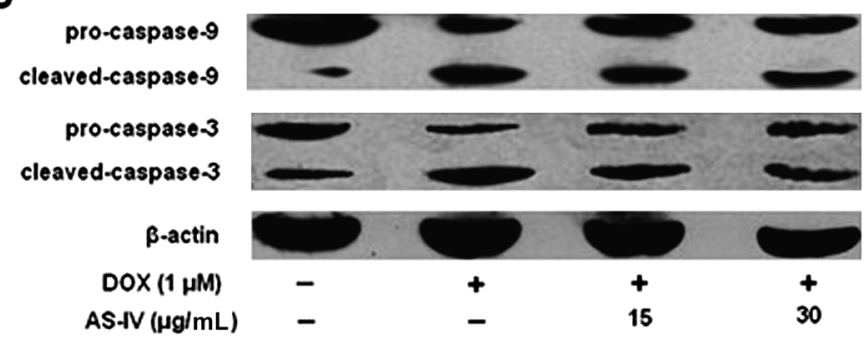

口cleaved-caspase-9/3-actin - cleaved-caspase-3/ß-actin

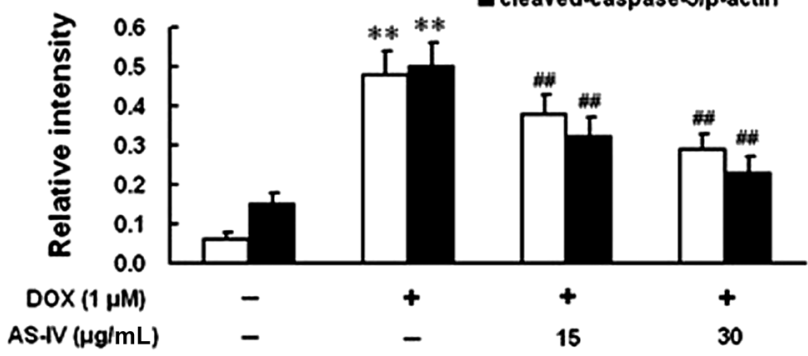

Fig. 6. Effects of AS-IV on the Expression Levels of Proteins Associated with the Mitochondrial Apoptotic Pathway in DOX-Treated Cardiomyocytes (Western Blot Analysis)

(A) Protein expression levels of Bcl-2, Bax and CytC. (B) Protein expression levels of cleaved-caspase-9 and cleaved-caspase-3. $\beta$-Actin was used as internal control. The protein expression levels of Bcl-2, Bax, CytC, cleaved-caspase- 9 and cleaved-caspase- 3 were normalized to that of $\beta$-actin. Data are presented as mean \pm S.D. $* * p<0.01 v s$. Control group; ${ }^{\#} p<0.01 v s$. DOX $(1 \mu \mathrm{M})$ group. All experiments were repeated at least three times.

A

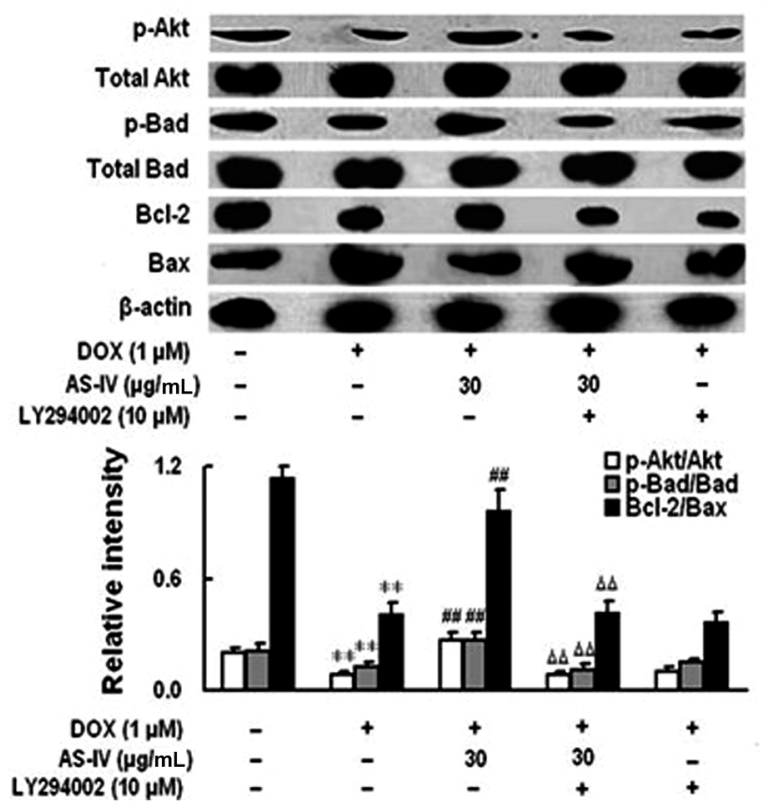

B

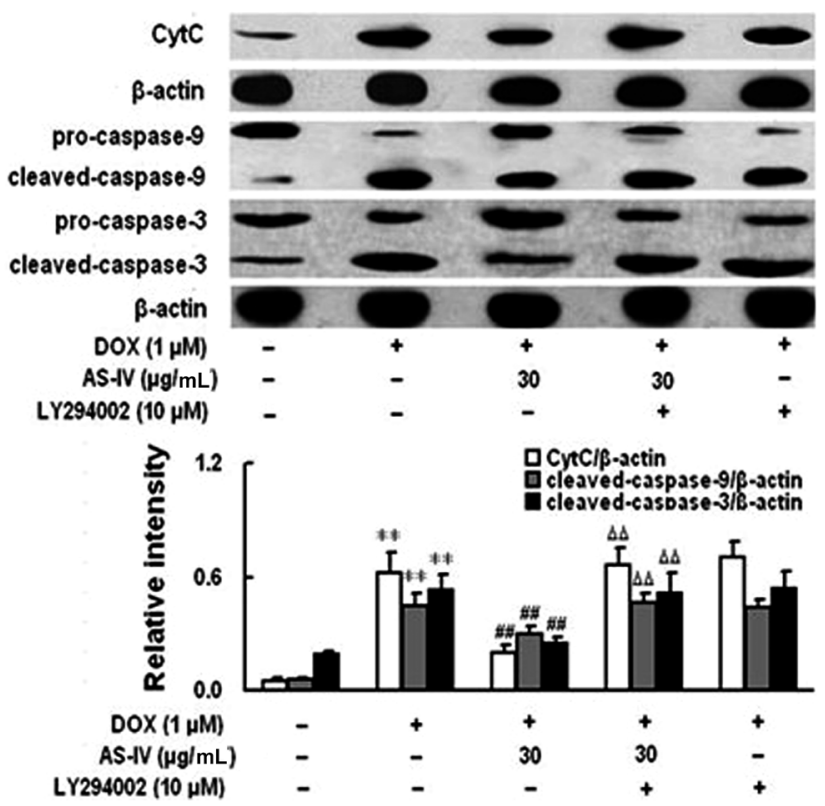

Fig. 7. Effects of AS-IV on the Expression Levels of Proteins Associated with the PI3K/Akt and Mitochondrial Apoptotic Pathways in DOX-Treated Cardiomyocytes (Western Blot Analysis)

(A) Protein expression levels of p-Akt, p-Bad, Bcl-2 and Bax. (B) Protein expression levels of CytC, cleaved-caspase-9 and cleaved-caspase-3. p-Akt/Akt, p-Bad/ $\mathrm{Bad}$ and $\mathrm{Bcl}-2 / \mathrm{Bax}$ were determined by densitometric quantification of each band. $\beta$-Actin was used as internal control. The protein expression levels of CytC, cleavedcaspase-9 and cleaved-caspase-3 were normalized to that of $\beta$-actin. Data are presented as mean \pm S.D. ${ }^{* *} p<0.01 v s$. Control group; ${ }^{\#} p<0.01 v s$. DOX (1 $\left.\mu \mathrm{M}\right)$ group; $\Delta \Delta p<0.01 v s$. DOX $(1 \mu \mathrm{m})+\mathrm{AS}-\mathrm{IV}(30 \mu \mathrm{g} / \mathrm{mL})$ group. All experiments were repeated at least three times.

protein and the release of $\mathrm{CytC}$, as compared with the $\mathrm{DOX}$ group ( $p<0.01$, Fig. 6A). Additionally, AS-IV significantly reduced the expressions of caspase-9 and caspase-3 proteins via inhibiting the proteolysis of pro-caspase- 9 and pro-caspase-3, respectively, as compared with the DOX group $(p<0.01$, Fig. 6B).

For further insight into the mechanism by which AS-IV regulated the expressions of $\mathrm{Bcl}-2$ and Bax proteins, the ex- pression levels of proteins associated with the PI3K/Akt pathway and MAP were both detected by western blot analysis (Fig. 7). As shown in Fig. 7A, DOX significantly reduced the phosphorylation of Akt and Bad and the expression of Bcl-2 protein, whereas markedly enhanced the expression of Bax protein and reduced the $\mathrm{Bcl}-2$ to Bax ratio, as compared with the Control group $(p<0.01)$. As shown in Fig. 7B, DOX markedly promoted the release of $\mathrm{CytC}$ and the sequential expres- 


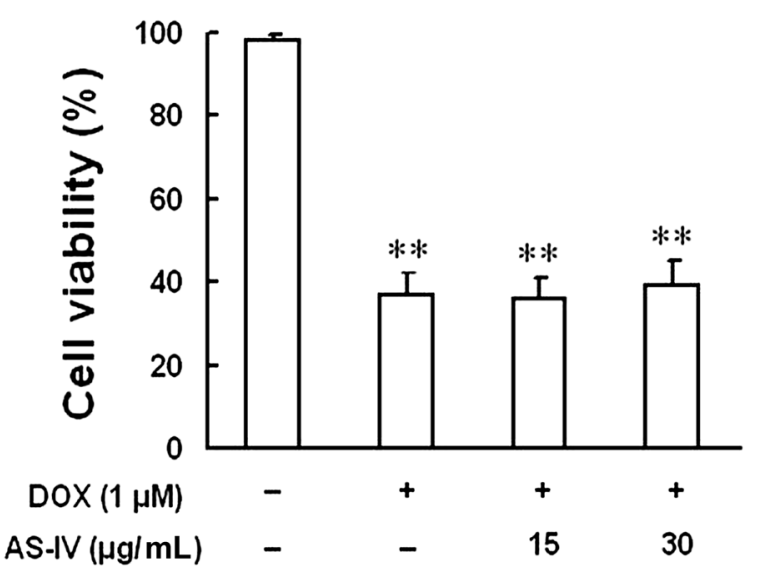

Fig. 8. Effect of AS-IV on the Chemotherapeutic Efficacy of DOX (MTT Assay)

Data are presented as mean \pm S.D. ${ }^{* *} p<0.01$ vs. Control group. All experiments were repeated at least three times.

sions of caspase- 9 and caspase- 3 proteins, as compared with the Control group $(p<0.01)$. However, AS-IV significantly enhanced the phosphorylation of Akt and Bad and the expression of $\mathrm{Bcl}-2$ protein, whereas markedly reduced the expression of Bax protein and restored the Bcl-2 to Bax ratio, as compared with the DOX group (Fig. 7A). In addition, AS-IV significantly reduced the release of $\mathrm{CytC}$ and the expressions of caspase- 9 and caspase-3 proteins, as compared with the DOX group ( $p<0.01$, Fig. 7B). Furthermore, the effects of AS-IV on the expressions of $\mathrm{Bcl}-2$ and Bax proteins were significantly inhibited by PI3K inhibitor LY294002 $(p<0.01)$. LY294002 treatment didn't affect the effects of DOX on the expressions of proteins aforementioned.

AS-IV Had No Effect on the Antitumor Activity of DOX To evaluate whether AS-IV could modify the chemotherapeutic efficacy of DOX, the effect of AS-IV on DOX-induced cell killing in MCF-7 human breast cancer cells was investigated by MTT assay. The results, as shown in Fig. 8, indicated that co-administration of DOX with AS-IV had no significant effect on DOX-induced cancer cell death.

\section{Discussion}

The new observations of this study, which provided further insight into the pathogenesis of DIC as well as casted new light on the cardioprotective effect of AS-IV, are showed below: (a) the present study is the first to characterize the protective effects and associated underlying mechanisms of AS-IV against DIC in vitro; (b) our study provides the first evidence that AS-IV significantly ameliorated the cytotoxicity, mitochondrial damage and dysfunction induced by DOX in cardiomyocytes; (c) for the first time, we found that DIC was caused by mitochondrial dysfunction as well as consequent cardiomyocyte apoptosis mediated by MAP via downregulation of Akt and Bad phosphorylation; (d) our data are the first documentation that the activation of PI3K/Akt pathway instimulated by AS-IV was an upstream and pivotal event in suppressing the cardiomyocyte apoptosis mediated by MAP in DIC; (e) most importantly, AS-IV didn't diminish the antitumor activity of DOX, which is also reported for the first time.

Cardiomyocyte loss and dysfunction are widely recognized as typical features of DIC. Based on this, firstly, we deter- mined the effect of AS-IV on DOX-induced cytotoxicity in cardiomyocytes. Consistent with the findings of previous studies, ${ }^{28,29)}$ our study showed that DOX significantly reduced the cTnI expression, a golden standard for cardiomyocyte death diagnosis, as well as the beating cell ratio and beating rate in cardiomyocytes. These changes, together with the markedly decreased viability, indicated the occurrence of severe cardiomyocyte loss and dysfunction. However, AS-IV significantly reduced these adverse changes elicited by DOX, suggesting that AS-IV attenuates DOX-induced cytotoxicity in cardiomyocytes, which is reported for the first time.

The mechanism for DIC, particularly for the cardiomyocyte cytotoxicity induced by DOX, has been widely believed to be attributable to the mitochondrial dysfunction caused by DOX-generated ROS, which then activate MAP leading to caspase-3 activation and cardiomyocyte apoptosis, ${ }^{9,30)}$ especially in immature cardiomyocytes. ${ }^{31)}$ ROS-caused mitochondrial dysfunction always leads to a tremendous decrease in ATP biosynthesis and a release of $\mathrm{CytC},{ }^{15,32)}$ which in turn further aggravates itself. This forms a catastrophic cycle of mitochondrial functional declination, leading to ATP depletion and ROS excess, which could induce Bax translocation from cytosol to mitochondria, resulting in the activation of MAP. ${ }^{17,19)}$ Based on the serious cytotoxicity observed and the notions mentioned above, we investigated the effects of AS-IV on DOX-induced mitochondrial toxicity. As a result, AS-IV substantially reduced the elevated mitochondrial ROS level, and $\mathrm{LDH}$ and $\mathrm{CK}-\mathrm{MB}$ activities in mitochondrial supernatant in DOX-treated cardiomyocytes, which are three important cardiac injury markers, suggesting that AS-IV attenuates the mitochondrial damage caused by DOX. It is well known that $\mathrm{SDH}$ is a component of complex II and plays a vital role in regulating mitochondrial respiratory activity, ${ }^{33)}$ and that ATP synthase is the most significant enzyme in ATP biosynthesis. As marker enzymes of mitochondrial function, significantly declined SDH and ATP synthase activities indicate the occurrence of mitochondrial dysfunction. ${ }^{34)}$ Furthermore, CytC release and ATP depletion are the most substantial evidences of mitochondrial dysfunction. ${ }^{15)}$ Consistent with the findings of earlier studies, ${ }^{15,25,34)}$ in this study, DOX caused significant mitochondrial dysfunction, as evidenced by the markedly reduced activities of SDH and ATP synthase and level of ATP, as well as a considerable increase in the release of CytC. However, AS-IV significantly inhibited the CytC release, and restored the ATP level via preservation of SDH and ATP synthase activities. Most importantly, AS-IV treatments alone markedly elevated the ATP synthase activity and ATP level in a dose-dependent manner. These findings highlight the specific mitochondrial protective effects of AS-IV in the context of DOX exposure for the first time.

Following that, the morphological features of apoptosis were identified by Hoechst 33258 staining, and the apoptosis rate was accurately calculated via flow cytometry. Taken together with the results of these two independent assays, it is apparent that DOX-induced cardiomyocyte apoptosis was significantly inhibited by AS-IV, which provides convictive evidences for the anti-apoptotic effect of AS-IV against DIC.

For further confirming our hypothesis, the expressions of proteins associated with MAP were detected. Accumulating evidence has shown that DOX can activate MAP via enhancing Bax expression and/or inhibiting Bcl-2 expression, which 
reduces the ratio of $\mathrm{Bcl}-2$ to $\mathrm{Bax}$, leading to the $\mathrm{CytC}$ release and sequential activation of caspase- 9 and caspase- $3 .{ }^{25,31)}$ Consistent with these findings, our western blot data showed same effects of DOX, indicating that it was the MAP that mediated the cardiomyocyte apoptosis observed. In contrast, AS-IV significantly restored the Bcl-2 to Bax ratio via enhancing Bcl-2 protein expression and inhibiting Bax protein expression, which is consistent with the findings of Li et al. ${ }^{23)}$ Our further investigations showed that the restoration of $\mathrm{Bcl}-2$ to Bax ratio markedly reduced the $\mathrm{CytC}$ release and sequential activation of caspase-9 and caspase-3, indicating that the inactivation of MAP was responsible for the anti-apoptotic effect of AS-IV against DIC, which was firstly found.

Then how is it possible for AS-IV to modulate the expressions of Bcl-2 and Bax proteins? The activation of the PI3K/ Akt pathway has been suggested responsible for AS-IV's anti-apoptotic effect. ${ }^{35)}$ Coincidentally, phosphorylation of Akt could phosphorylate Bad at Ser136 to make it depolymerize with Bcl-2, which then increases Bcl-2 activity leading to depressed caspase activities and cell survival. ${ }^{36)}$ In addition, it has been found that phosphorylation of Akt could also reduce Bax activity via suppressing Bax translocation from cytosol to mitochondria. ${ }^{37)}$ Most importantly, DOX-induced cardiomyocyte apoptosis has been associated with downregulation of Akt phosohorylation in vitro and in vivo, and suppressed by the phosphorylation of Akt and Bad. ${ }^{38-40)}$ Based on these, we speculated that PI3K/Akt/Bad signaling pathway was responsible for AS-IV's modulatory effects on Bcl-2 and Bax protein expressions. In this study, DOX significantly reduced the phosphorylation levels of Akt and Bad, leading to a substantial decrease in the Bcl-2 protein expression. This change, together with the enhanced Bax protein expression, activated the MAP. However, AS-IV significantly elevated Akt and Bad phosphorylation levels, resulting in a significant increase in the Bcl-2 protein expression and a considerable decrease in the Bax protein expression, which evidently reduced the release of CytC and activation of caspase- 9 and caspase- 3 . For further confirming the participation of PI3K/Akt pathway in inactivating the MAP, the PI3K inhibitor LY294002 was used. $^{26)}$ As a result, the effects of AS-IV on the expressions of p-Akt, p-Bad, Bcl-2 and Bax proteins, release of $\mathrm{CytC}$, as well as activation of caspase- 9 and caspase-3 were all significantly inhibited by LY294002. Most importantly, the inhibitory effect of LY294002 on the cardioprotection of AS-IV showed in the western blot analysis was in line with those observed in independent assays, including the MTT assay, Hoechst 33258 staining and flow cytometry. These findings firstly and convictively confirmed that it was the activation of PI3K/Akt pathway that suppressed the cardiomyocyte apoptosis mediated by MAP in AS-IV-induced survival signaling in cardiomyocytes.

Finally, we determined the effect of AS-IV on the antitumor activity of DOX. The primary goal of DOX therapy is to kill cancer cells or inhibit their proliferation. If the drug used for attenuating DIC significantly interferes with the antitumor efficacy of DOX, this would compromise the value of such adjunct treatment. Hence, it is vitally necessary to detect the effect of such drug on the antitumor effect of DOX. Our results firstly showed that AS-IV didn't affect DOX-induced cell killing in MCF-7 human breast cancer cells, suggesting that AS-IV has no interference on the antineoplastic activity of DOX.
In conclusion, this study suggests that AS-IV holds promise as an efficient intervention for preventing DIC. The understanding of the exactly modulatory mechanisms of AS-IV on DIC will be helpful to reduce and/or even avoid DIC in cancer patients with DOX chemotherapy. Further studies are now in progress.

Acknowledgment The authors are grateful to Dr. Yihui Deng for his excellent assistance.

\section{References}

1) Singal P. K., Siveski-Iliskovic N., Hill M., Tomas T. P., Li T., J. Mol. Cell. Cardiol., 27, 1055-1063 (1995).

2) Singal P. K., Li T., Kumar D., Danelisen I., Iliskovic N., Mol. Cell. Biochem., 207, 77-86 (2000).

3) Li T., Danelisen I., Singal P. K., Mol. Cell. Biochem., 232, 19-26 (2002).

4) Wouters K. A., Kremer L. C., Miller T. L., Herman E. H., Lipshultz S. E., Br. J. Haematol., 131, 561-578 (2005).

5) van Dalen E. C., Caron H. N., Dickinson H. O., Kremer L. C., Cochrane Database Syst. Rev., 6, CD003917 (2011).

6) Lebrecht D., Kokkori A., Ketelsen U. P., Setzer B., Walker U., J. Pathol., 207, 436-444 (2005).

7) Shi J., Abdelwahid E., Wei L., Curr. Pediatr. Rev., 7, 329-336 (2011).

8) Doroshow J. H., Davies K. J., J. Biol. Chem., 261, 3068-3074 (1986).

9) Kalyanaraman B., Joseph J., Kalivendi S., Wang S., Konorev E., Kotamraju S., Mol. Cell. Biochem., 234/235, 119-124 (2002).

10) von Harsdorf R., Li P. F., Dietz R., Circulation, 99, 2934-2941 (1999).

11) Youn H. J., Kim H. S., Jeon M. H., Lee Ju. H., Seo Y. J., Lee Y. J., Lee Je. H., Mol. Cell. Biochem., 270, 13-19 (2005).

12) Poizat C., Puri P. L., Bai Y., Kedes L., Mol. Cell. Biol., 25, $2673-$ 2687 (2005).

13) Wallace K. B., Cardiovasc. Toxicol., 7, 101-107 (2007).

14) Ohkura K., Lee J. D., Shimizu H., Nakano A., Uzui H., Horikoshi M., Fujibayashi Y., Yonekura Y., Ueda T., Mol. Cell. Biochem., 248 , 203-208 (2003)

15) Berthiaume J. M., Wallace K. C., Cell Biol. Toxicol., 23, 15-25 (2007).

16) Zhang Y. W., Shi J., Li Y. J., Wei L., Arch. Immunol. Ther. Exp. (Warsz.), 57, 435-445 (2009).

17) Green P. S., Leeuwenburgh C., Biochim. Biophys. Acta, 1588 94-101 (2002).

18) Chen M. H., Kerkelä R., Force T., Circulation, 118, 84-95 (2008).

19) Chen X. J., Meng D., Feng L., Bian Y. Y., Li P., Yang D., Cao K. J. Zhang J. N., Am. J. Chin. Med., 34, 1015-1025 (2006).

20) Li Z. P., Cao Q., Acta Pharmacol. Sin., 23, 898-904 (2002).

21) Guan F. Y., Li H., Sun W., Yang S. J., Journal of Jilin University, 33, 211-214 (2007), Medicine Edition.

22) Zhong F., Du J. Z., Yu X. H., Zhang Z. C., Chen R. Z., Li S. J., Journal of University of South China, 32, 17-19 (2004).

23) Li L., Tao H. Y., Chen J. B., Zhongguo Zhong Xi Yi Jie He Za Zhi, 26, 1011-1014 (2006).

24) Chlopcíková S., Psotová J., Miketová P., Biomed. Pap. Med. Fac. Univ. Palacky Olomouc Czech Repub., 145, 49-55 (2001).

25) Clause K. C., Tchao J., Powell M. C., Liu L. J., Huard J., Keller B. B., Tobita K., PLoS One, 7, e40725 (2012).

26) Lv X., Yu X., Wang Y., Wang F., Li H., Wang Y., Lu D., Qi R., Wang H., PLoS ONE, 7, e47351 (2012).

27) Li C., Tian J., Li G., Jiang W., Xing Y., Hou J., Zhu H., Xu H., Zhang G., Liu Z., Ye Z., Eur. J. Pharmacol., 649, 100-107 (2010).

28) Bian Y., Sun M., Silver M., Ho K. K., Marchionni M. A., Caggiano A. O., Stone J. R., Amende I., Hampton T. G., Morgan J. P., Yan X., 
Am. J. Physiol. Heart Circ. Physiol., 297, H1974-H1983 (2009).

29) Takahashi K., Fujita Y., Mayumi T., Hama T., Kishi T., Chem. Pharm. Bull., 35, 326-334 (1987).

30) Octavia Y., Tocchetti C. G., Gabrielson K. L., Janssens S., Crijns H. J., Moens A. L., J. Mol. Cell. Cardiol., 52, 1213-1225 (2012).

31) Simůnek T., Stérba M., Popelová O., Adamcová M., Hrdina R., Gersl V., Pharmacol. Rep., 61, 154-171 (2009).

32) Štěrba M., Popelová O., Lenčo J., Fučíková A., Brčáková E., Mazurová Y., Jirkovský E., Simůnek T., Adamcová M., Mičuda S., Stulík J., Geršl V., J. Mol. Cell. Cardiol., 50, 849-862 (2011).

33) Xu L. J., Jin L., Pan H., Zhang A. Z., Wei G., Li P. P., Lu W. Y., Acta Pharmacol. Sin., 27, 1333-1339 (2006).

34) Lê-Quôc K., Lê-Quôc D., Gaudemer Y., Biochemistry, 20, $1705-$ 1710 (1981)
35) Zhu F. F., Yin Y. Y., Li W. P., Li W. Z., Wu G. C., Gong H. L., Chinese Pharmacological Bulletin, 25, 213-216 (2009).

36) Fan G. C., Zhou X., Wang X., Song G., Qian J., Nicolaou P., Chen G., Ren X., Kranias E. G., Circ. Res., 103, 1270-1279 (2008).

37) Raphael J., Abedat S., Rivo J., Meir K., Beeri R., Pugatsch T., Zuo Z., Gozal Y., J. Pharmacol. Exp. Ther., 318, 186-194 (2006).

38) Park J. E., Kang Y. J., Park M. K., Lee Y. S., Kim H. J., Seo H. G., Lee J. H., Hye Sook Y. C., Shin J. S., Lee H. W., Ahn S. K., Chang K. C., Int. Immunopharmacol., 6, 226-233 (2006).

39) Tsuruta F., Masuyama N., Gotoh Y., J. Biol. Chem., 277, 1404014047 (2002).

40) Li K., Sung R. Y., Huang W. Z., Yang M., Pong N. H., Lee S. M., Chan W. Y., Zhao H., To M. Y., Fok T. F., Li C. K., Wong Y. O., Ng P. C., Circulation, 113, 2211-2220 (2006). 